\title{
Biodiversity and monthly density fluctuations of water mites in Khankra gad, a spring-fed tributary of river Alaknanda, Pauri Garhwal in Uttarakhand, India
}

\section{Shailza Negi}

Ecology Lab, Department of Zoology, HNB Garhwal University (A Central University), BGR Campus, Pauri Garhwal-246001 (Uttarakhand), India

\section{A.K. Dobriyal}

Ecology Lab, Department of Zoology, HNB Garhwal University (A Central University), BGR Campus, Pauri Garhwal-246001 (Uttarakhand), India

Pankaj Bahuguna*

Aquatic Biodiversity Lab, Department of Zoology, B.D. Govt. P.G. college, Jaihrikhal, Pauri Garhwal-246193 (Uttarakhand), India

*Corresponding author. Email: pankajpaurii@gmail.com

\section{Article Info}

https://doi.org/10.31018/ jans.v13i1.2568 Received: February 3, 2021 Revised: March 5, 2021 Accepted: March 7, 2021

\section{How to Cite}

Negi, S. et al. (2021). Biodiversity and monthly density fluctuations of water mites in Khankra gad, a spring-fed tributary of river Alaknanda, Pauri Garhwal in Uttarakhand, India . Journal of Applied and Natural Science, 13(1): 258 - 267. https:// doi.org/10.31018/jans.v13i1.2568

\begin{abstract}
Hydrachnidia is an important group of aquatic invertebrates. They play an important role in regulating other invertebrate populations, thus influencing the composition and functionality of river ecosystems. The present study aims to assess the habitat ecology, density and diversity of aquatic mites in the Khankra gad, Rudraprayag district for a period of two year on a monthly basis, from July 2018 to June 2020. The Khankra gad is a perennial spring-fed stream originating from the Bansoun peak in district-Rudraprayag of Garhwal Himalaya ( $800 \mathrm{~m}$ asl). A total of 2537 Hydrachnidia samples were collected, belonging to 6 families viz, Torrenticolidae, Sperchontidae, Feltriidae, Hygrobatidae, Lebertiidae and Aturidae. Sperchontidae, Torrenticolidae and Hygrobatidae were the common families recorded in both spots, whereas Feltriidae was recorded in Spot-1, Lebertiidae and Aturidae were recorded in Spot-2. The highest numbers (1842) of Hydrachnidia were collected from Spot-2. A total of 19 aquatic mite species were recorded in Spot-1 and 25 species in Spot-2 throughout the study period. Aquatic mites showed maximum density (177 units. $\mathrm{m}^{-2}$ in Spot-1 and 274 units. $\mathrm{m}^{-2}$ in Spot-2) in December and minimum (11 units. $\mathrm{m}^{-2}$ in Spot-1 and 17 units. $\mathrm{m}^{-2}$ in Spot-2) in July. Various ecological parameters of our study indicated that Khankra gad is a good habitat for aquatic mites.
\end{abstract}

Keywords: Water mites, Density, Diversity, Habitat ecology, Uttarakhand

\section{INTRODUCTION}

Hydrachnidia, commonly called water mites, is among the most diverse freshwater Acari groups, which are widely neglected because of their small size (Cook and Mitchell 1953). In aquatic ecosystems, mites are important for maintaining the food web as they feed on many invertebrate eggs and larvae such as Diptera, Trichoptera, Plecoptera, Odonata and others (Martin 2008). Walter (1928) and Lundblad (1934) were the pioneer in publishing records of Hydrachnidia fauna from the Indian Himalaya. Kumar and Dobriyal (1992) studied the water mite fauna from Garhwal streams for the first time. A significant contribution to taxonomy of hill stream water mites of the Garhwal region have been made by Kumar et al. (2006, 2007), Pesic et al. (2007a,b), Pesic and Panesar (2008), Pesic et al. (2012), Pesic et al., (2019a,b) and Pesic et al., (2020a,b). Bahuguna et al. (2019 for spring-fed water; 2020-for glacier-fed water) carried out extensive work on the hill stream mite species' density and diversity. The 30 water mite species have been reported from Garhwal region so far (Bahuguna and Negi, 2020). Later Bahuguna and Dobriyal (2020) gave a detailed analysis of population structure and drifting pattern on water mites from Garhwal Himalaya, Uttarakhand, In- 
dia.

The present study is being conducted in the Khankra gad stream of Garhwal Himalaya. The purpose of the study was to analyze the water mite community's structure through the indices of richness and regularity to determine the density and diversity of Hydrachnidia species with respect to the physicochemical parameters of July 2018 to June 2020.

\section{MATERIALS AND METHODS}

\section{Ethical statement}

There is no ethical issue in this study, as work was conducted on aquatic insects (water mites).

\section{Study area}

Khankra gad is a $3^{\text {rd }}$ order spring-fed stream of Garhwal Himalaya (Fig.1). The stream originates from the Bansoun peak of Garhwal Himalaya. It is based in the
Khankra village of Rudraprayag district in Uttarakhand (800 $\mathrm{m}$ asl). Two sampling stations were selected, Spot $-1 \quad\left(30^{\circ} 14^{\prime} 37.12^{\prime \prime} \mathrm{N}\right.$ and $\left.78^{\circ} 55^{\prime} 06.00^{\prime \prime} \mathrm{E}\right)$ situated upstream at an altitude of 723 masl and Spot-2 $\left(30^{\circ}\right.$ $14 ' 45.76 " \mathrm{~N}$ and $\left.78^{\circ} 54^{\prime} 55.84 " \mathrm{E}\right)$ situated downstream at an altitude of $670 \mathrm{~m}$ asl. Riparian vegetation in this study area play an important role in providing shelter and shade to regulate temperature.

\section{Laboratory work}

For the analysis of the physicochemical parameters, water samples were taken monthly for a period of two year from July 2018 to June 2020, and they were analyzed on the spot using methods as described in Welch (1948) and APHA (2012). Water temperature was measured by a centigrade thermometer and current velocity by float method. $\mathrm{pH}$ was measured by using a portable $\mathrm{pH}$ meter and DO by the Winkler method. Monthly samples of mites were taken very carefully

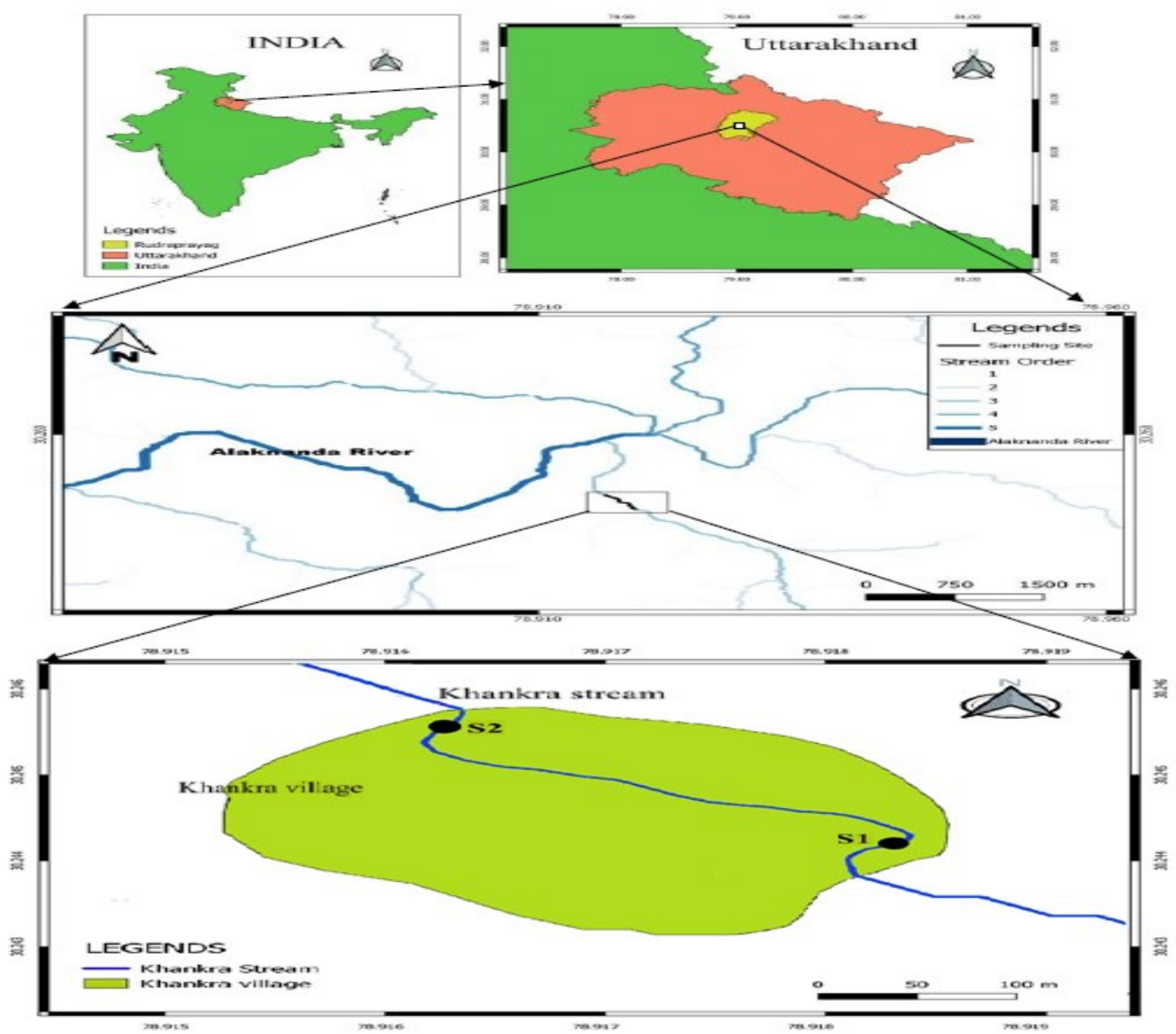

Fig 1. Sampling locations (Spot-1 and Spot-2) in Khankra gad, a spring-fed tributary of river Alaknanda, Uttarakhand, India. 
Negi, S. et al. / J. Appl. \& Nat. Sci. 13(1): 258 - 267 (2021)

from different habitats (submerged vegetation, pebbles and cobbles) using soft brushes in the stream or using a hand net from the stream bed. Samples of water mites were brought to the laboratory and transferred from $70 \%$ ethanol to Koenike fluid. Further processing was performed according to the standard methodology (Mitchell and Cook 1952). Samples were separated and examined under a stereomicroscope. The species were identified using various keys provided by Cook (1967, 1974), Prasad (1974), Gerecke (2003), Kumar et al. (2007), Pesic and Panesar (2008), Pesic et al. (2019a, b) and Pesic et al.,2020 a, b).

\section{Statistical data analysis}

The Margalef species richness index (d) was used to determine species richness (Margalef, 1958), Shannon diversity index $\left(\mathrm{H}^{\prime}\right)$ calculated to determine species diversity in a community by using Shannon- Wiener diversity index (1949), evenness of species in a community is calculated by Pielou's index ( $\mathrm{J}$ ) (Pielou, 1966), The Simpson index (D) was calculated to determine the dominant species (Edward H. Simpson, 1949). The similarity of species was calculated by using cluster analysis. Pearson correlation analysis and linear regression plots were also calculated to determine the relationship between water mite species abundance and physicochemical parameters of water. Statistical analysis was performed using PAST software version 3.16.

\section{RESULTS}

The results of habitat ecology are presented in Table 1 and Table 2 for Spot-1 and Spot-2, respectively. $\mathrm{pH}$ of the two sampling stations ranged from $7.4 \pm 0.1$ to
$7.9 \pm 0.1$ for Spot-1 and $9.2 \pm 0.2$ to $7.8 \pm 0.3$ for Spot-2. The water temperature ranged from $7.6 \pm 0.1$ to $12.8 \pm 0.2$ for Spot-1 and $9.7 \pm 0.2$ to $18.4 \pm 0.8$ for Spot2. Dissolved oxygen was maximum at Spot-1(8.8 \pm 0.3 mg. $\left.\mathrm{l}^{-1}\right)$ and at least at Spot-2 $\left(7.2 \pm 0.1 \mathrm{mg} . \mathrm{I}^{-1}\right)$. Stream

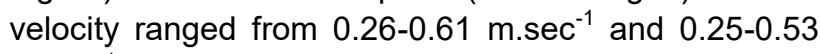
m.sec ${ }^{-1}$ at Spot-1 and Spot-2 respectively. Total maximum alkalinity was $99.1 \mathrm{mg.l}^{-1}$ at Spot-1 in January and at Spot-2 $99.9 \mathrm{mg.l}^{-1}$ in March. Total hardness was maximum in January for both the spots, $121.91{\mathrm{mg} . \mathrm{I}^{-1}}^{-1}$ in Spot-1 and 109.8 in Spot-2 (Table 1 and Table 2).

The abundance and diversity of water mites from the sampling Spot-1 and Spot-2 are summarized in Tables 3 and 4 , respectively. There was an increase in the diversity of water mites at Spot-2 in comparison to Spot -1 . Spot-2 is situated at an altitude of 670 masl where the maximum diversity of mites (25 species) was observed. Spot-1, at an altitude of 723 masl had 19 species (Table 3 and 4).

A total of 2504 Hydrachnidia samples were collected from two sampling spots from July 2018 to Jun 2020, belonging to 6 families viz, Torrenticolidae, Sperchontidae, Feltriidae, Hygrobatidae, Lebertiidae, and Aturidae. A total of 19 species were observed in Spot-1, belonging to four families, viz., Torrenticolidae, Sperchontidae, Feltriidae, Hygrobatidae and 25 species were recorded from Spot-2, belonging to 5 families viz., Torrenticolidae, Sperchontidae, Hygrobatidae, Lebertiidae and Aturidae (Table 3 and Table 4). Sperchontidae, Torrenticolidae and Hygrobatidae were the common families recoded in both spots, whereas Feltriidae was recorded in Spot-1, Lebertiidae and Aturidae were recorded from Spot-2.

From Spot-1, a maximum density of 177 units. $\mathrm{m}^{-2}$ was recorded in January and a minimum 11 units. $\mathrm{m}^{-2}$ in

Table 1. Average values of physicochemical parameters of the Khankra gad at Spot-1 during 2018-20.

\begin{tabular}{|c|c|c|c|c|c|c|c|}
\hline $\begin{array}{l}\text { Month } \\
(2018-2020)\end{array}$ & $\begin{array}{l}\text { WT } \\
\left({ }^{0} \mathrm{C}\right)\end{array}$ & $\mathrm{pH}$ & $\begin{array}{l}\text { CV } \\
\left(\mathrm{m} . \mathrm{sec}^{-1}\right)\end{array}$ & $\begin{array}{l}\text { DO } \\
\left(\mathrm{mg} \cdot \mathrm{I}^{-1}\right)\end{array}$ & $\begin{array}{l}\text { TA } \\
\left(\mathbf{m g} \cdot \mathrm{I}^{-1}\right)\end{array}$ & $\begin{array}{l}\text { TH } \\
\left(\mathbf{m g} \cdot \mathrm{I}^{-1}\right)\end{array}$ & $\begin{array}{l}\text { Fee } \mathrm{CO}_{2} \\
\left(\mathrm{mg} \cdot \mathrm{I}^{-1}\right)\end{array}$ \\
\hline July & $12.4 \pm 0.2$ & $7.4 \pm 0.1$ & $0.61 \pm 0.01$ & $7.3 \pm 0.1$ & $81.2 \pm 0.8$ & $82 \pm 0.3$ & Nil \\
\hline August & $12.8 \pm 0.2$ & $7.5 \pm 0.3$ & $0.57 \pm 0.01$ & $7.7 \pm 0.1$ & $81.7 \pm 1.7$ & $90.2 \pm 0.3$ & Nil \\
\hline September & $11.3 \pm 0.3$ & $7.7 \pm 0.1$ & $0.43 \pm 0.02$ & $8.3 \pm 0.2$ & $82.7 \pm 1.4$ & $91.5 \pm 1.9$ & Nil \\
\hline October & $10.8 \pm 0.3$ & $7.5 \pm 0.1$ & $0.35 \pm 0.01$ & $8.5 \pm 0.2$ & $85.1 \pm 0.8$ & $105.5 \pm 2.7$ & Nil \\
\hline November & $8.7 \pm 0.2$ & $7.6 \pm 0.1$ & $0.32 \pm 0.01$ & $8.7 \pm 0.3$ & $87.8 \pm 2.9$ & $113.8 \pm 3.4$ & Nil \\
\hline December & $8.5 \pm 0.3$ & $7.9 \pm 0.1$ & $0.3 \pm 0.01$ & $8.8 \pm 0.3$ & $91.4 \pm 1.6$ & $118.5 \pm 1.9$ & Nil \\
\hline January & $7.6 \pm 0.1$ & $7.8 \pm 0.3$ & $0.26 \pm 0.02$ & $8.7 \pm 0.1$ & $99.1 \pm 1.1$ & $121.9 \pm 6.2$ & Nil \\
\hline February & $8.2 \pm 0.3$ & $7.6 \pm 0.2$ & $0.29 \pm 0.01$ & $8.1 \pm 0.1$ & $97.5 \pm 0.8$ & $108.6 \pm 1.5$ & Nil \\
\hline March & $8.5 \pm 0.1$ & $7.5 \pm 0.2$ & $0.31 \pm 0.02$ & $8 \pm 0.2$ & $96 \pm 1.1$ & $105.5 \pm 1.8$ & Nil \\
\hline April & $9.7 \pm 0.2$ & $7.7 \pm 0.3$ & $0.35 \pm 0.01$ & $7.8 \pm 0.1$ & $93.9 \pm 1.2$ & $10.2 \pm 0.8$ & Nil \\
\hline May & $10.2 \pm 0.1$ & $7.5 \pm 0.2$ & $0.37 \pm 0.02$ & $8.1 \pm 0.1$ & $90.8 \pm 0.8$ & $99.7 \pm 0.7$ & Nil \\
\hline June & $10.7 \pm 0.2$ & $7.7 \pm 0.1$ & $0.39 \pm 0.02$ & $7.8 \pm 0.1$ & $89 \pm 0.6$ & $95.4 \pm 1.3$ & Nil \\
\hline
\end{tabular}


Negi, S. et al. / J. Appl. \& Nat. Sci. 13(1): 258 - 267 (2021)

Table 2. Average values of physicochemical parameters of the Khankra gad at Spot-2 during 2018-20.

\begin{tabular}{|c|c|c|c|c|c|c|c|}
\hline $\begin{array}{l}\text { Month } \\
(2018-2020)\end{array}$ & $\begin{array}{l}\text { WT } \\
\left(^{0} \mathrm{C}\right)\end{array}$ & $\mathrm{pH}$ & $\begin{array}{l}\text { CV } \\
\left(\mathrm{m} . \mathrm{sec}^{-1}\right)\end{array}$ & $\begin{array}{l}\text { DO } \\
\left(\mathrm{mg} . \mathrm{I}^{-1}\right)\end{array}$ & $\begin{array}{l}\text { TA } \\
\left(\mathrm{mg} . \mathrm{I}^{-1}\right)\end{array}$ & $\begin{array}{l}\text { TH } \\
\left(\mathrm{mg} . \mathrm{I}^{-1}\right)\end{array}$ & $\begin{array}{l}\text { Fee } \mathrm{CO}_{2} \\
\left(\mathrm{mg} \cdot \mathrm{I}^{-1}\right)\end{array}$ \\
\hline July & $18.4 \pm 0.8$ & $7.4 \pm 0.3$ & $0.53 \pm 0.01$ & $7.2 \pm 0.1$ & $75.7 \pm 0.8$ & $85.4 \pm 0.3$ & Nil \\
\hline August & $17.5 \pm 0.2$ & $7.3 \pm 0.2$ & $0.41 \pm 0.01$ & $7.4 \pm 0.1$ & $78.9 \pm 0.9$ & $85.2 \pm 1.4$ & Nil \\
\hline September & $17.4 \pm 0.3$ & $7.3 \pm 0.1$ & $0.35 \pm 0.01$ & $8 \pm 0.1$ & $81.5 \pm 1$ & $87.9 \pm 0.6$ & Nil \\
\hline October & $14 \pm 0.3$ & $7.5 \pm 0.2$ & $0.31 \pm 0.01$ & $8.1 \pm 0.3$ & $82.8 \pm 0.9$ & $93.3 \pm 0.6$ & Nil \\
\hline November & $11.3 \pm 0.3$ & $7.7 \pm 0.3$ & $0.28 \pm 0.01$ & $8.3 \pm 0.1$ & $85.9 \pm 1.4$ & $99 \pm 1.3$ & Nil \\
\hline December & $10.7 \pm 0.2$ & $7.8 \pm 0.3$ & $0.27 \pm 0.01$ & $8.3 \pm 0.1$ & $89.8 \pm 1.3$ & $108.1 \pm 0.8$ & Nil \\
\hline January & $9.7 \pm 0.2$ & $7.6 \pm 0.2$ & $0.25 \pm 0.01$ & $8.4 \pm 0.1$ & $94.7 \pm 1.3$ & $109.8 \pm 13.2$ & Nil \\
\hline February & $10.4 \pm 0.2$ & $7.5 \pm 0.1$ & $0.28 \pm 0.01$ & $7.7 \pm 0.2$ & $92.9 \pm 0.4$ & $102.7 \pm 1.4$ & Nil \\
\hline March & $11.8 \pm 0.3$ & $7.2 \pm 0.2$ & $0.29 \pm 0.01$ & $7.6 \pm 0.1$ & $99.9 \pm 0.5$ & $98.5 \pm 1.3$ & Nil \\
\hline April & $13.5 \pm 0.2$ & $7.2 \pm 0.1$ & $0.31 \pm 0.01$ & $7.4 \pm 0.1$ & $90.4 \pm 0.4$ & $93.9 \pm 0.5$ & Nil \\
\hline May & $15.7 \pm 0.1$ & $7.4 \pm 0.1$ & $0.33 \pm 0.01$ & $7.6 \pm 0.1$ & $89 \pm 0.6$ & $89 \pm 1.3$ & Nil \\
\hline June & $17.1 \pm 0.1$ & $7.6 \pm 0.1$ & $0.36 \pm 0.01$ & $7.3 \pm 0.1$ & $84.8 \pm 0.8$ & $85.6 \pm 1.3$ & Nil \\
\hline
\end{tabular}

Table 3. Monthly average variation of density and diversity of water mites in Khankra gad 2018-20 (Spot-1).

\begin{tabular}{|c|c|c|c|c|c|c|c|c|c|c|c|c|c|}
\hline $\begin{array}{l}\text { S. } \\
\text { No }\end{array}$ & Family/Genus/Species & Jul & Aug & Sep & Oct & Nov & Dec & Jan & Feb & Mar & Apr & May & Jun \\
\hline A & $\begin{array}{l}\text { F - Torrenticolidae Piersig } 1902 \\
\text { G - Torrenticola Piersig }\end{array}$ & & & & & & & & & & & & \\
\hline 1 & Torrenticola uttarakhandensis & 03 & 02 & 05 & 04 & 10 & 12 & 17 & 10 & 09 & 07 & 04 & 01 \\
\hline 2 & Torrenticola wonchoeli & 00 & 00 & 02 & 00 & 05 & 00 & 09 & 06 & 04 & 00 & 00 & 01 \\
\hline 3 & Torrenticola nana & 02 & 01 & 00 & 04 & 01 & 04 & 09 & 00 & 02 & 00 & 00 & 00 \\
\hline 4 & Torrenticola kumari & 01 & 00 & 01 & 00 & 02 & 06 & 07 & 04 & 00 & 03 & 05 & 03 \\
\hline 5 & Torrenticola semisuta & 00 & 00 & 00 & 01 & 00 & 06 & 11 & 03 & 00 & 04 & 00 & 00 \\
\hline 6 & $\begin{array}{l}\text { Torrenticola muranyii } \\
\text { G- Monatractides }\end{array}$ & 00 & 02 & 00 & 02 & 00 & 04 & 08 & 00 & 03 & 01 & 00 & 00 \\
\hline 7 & Monatractides garhwaliensis & 01 & 03 & 00 & 04 & 09 & 11 & 17 & 05 & 06 & 00 & 04 & 02 \\
\hline 8 & Monatractides oxystomus & 00 & 00 & 01 & 00 & 04 & 00 & 07 & 00 & 00 & 03 & 00 & 00 \\
\hline \multirow[t]{2}{*}{9} & Monatractides kontschani & 00 & 01 & 00 & 02 & 04 & 00 & 05 & 03 & 00 & 03 & 00 & 01 \\
\hline & Total & 07 & 09 & 09 & 17 & 35 & 43 & 90 & 31 & 24 & 21 & 13 & 08 \\
\hline B & $\begin{array}{l}\text { F - Sperchontidae Thor, } 1900 \\
\text { G - Sperchon Kramer }\end{array}$ & & & & & & & & & & & & \\
\hline 10 & Sperchon indicus & 03 & 05 & 07 & 09 & 11 & 14 & 21 & 16 & 11 & 09 & 07 & 02 \\
\hline 11 & Sperchon garhwalensis & 00 & 01 & 04 & 07 & 09 & 11 & 15 & 09 & 08 & 06 & 02 & 03 \\
\hline \multirow[t]{2}{*}{12} & Sperchon ootacamundis & 00 & 00 & 01 & 00 & 01 & 02 & 08 & 00 & 01 & 00 & 00 & 00 \\
\hline & Total & 03 & 06 & 12 & 16 & 21 & 27 & 44 & 25 & 20 & 15 & 09 & 05 \\
\hline C & $\begin{array}{l}\text { F - Hygrobatidae } \\
\text { G - Atractides Koch }\end{array}$ & & & & & & & & & & & & \\
\hline 13 & Atractides indicus & 01 & 05 & 04 & 07 & 09 & 12 & 17 & 09 & 04 & 05 & 03 & 02 \\
\hline 14 & Atractides garhwali & 00 & 03 & 00 & 07 & 06 & 09 & 14 & 08 & 04 & 03 & 00 & 00 \\
\hline 15 & Atractides incertus & 00 & 01 & 00 & 04 & 01 & 03 & 04 & 00 & 00 & 02 & 03 & 00 \\
\hline 16 & Atractides ootacamundis (Cook) & 00 & 00 & 01 & 02 & 00 & 00 & 01 & 00 & 03 & 00 & 01 & 00 \\
\hline 17 & $\begin{array}{l}\text { G - Hygrobates Koch } \\
\text { Hygrobates fluviatilis } \\
\text { Total }\end{array}$ & $\begin{array}{l}00 \\
01\end{array}$ & $\begin{array}{l}00 \\
09\end{array}$ & $\begin{array}{l}03 \\
08\end{array}$ & $\begin{array}{l}00 \\
20\end{array}$ & $\begin{array}{l}01 \\
17\end{array}$ & $\begin{array}{l}02 \\
26\end{array}$ & $\begin{array}{l}03 \\
39\end{array}$ & $\begin{array}{l}00 \\
17\end{array}$ & $\begin{array}{l}01 \\
12\end{array}$ & $\begin{array}{l}00 \\
10\end{array}$ & $\begin{array}{l}01 \\
08\end{array}$ & $\begin{array}{l}01 \\
03\end{array}$ \\
\hline D & $\begin{array}{l}\text { F- Feltriidae K.Viets, } 1926 \\
\text { G - Feltria Koenike, } 1892\end{array}$ & & & & & & & & & & & & \\
\hline 18 & Feltria gereckei & 00 & 00 & 00 & 01 & 00 & 00 & 02 & 01 & 00 & 03 & 00 & 01 \\
\hline \multirow[t]{3}{*}{19} & Feltria indica & 00 & 00 & 02 & 01 & 00 & 00 & 02 & 01 & 00 & 00 & 00 & 00 \\
\hline & Total & 00 & 00 & 02 & 02 & 00 & 00 & 04 & 02 & 00 & 03 & 00 & 01 \\
\hline & Total no. of water mites & 11 & 24 & 31 & 55 & 73 & 97 & 177 & 75 & 56 & 49 & 30 & 17 \\
\hline
\end{tabular}


Negi, S. et al. / J. Appl. \& Nat. Sci. 13(1): 258 - 267 (2021)

Table 4. Monthly average variation of density and diversity of water mites in Khankra gad 2018-20 (Spot-2).

\begin{tabular}{|c|c|c|c|c|c|c|c|c|c|c|c|c|c|}
\hline $\begin{array}{l}\text { S. } \\
\text { No. }\end{array}$ & Family/Genus/Species & Jul & Aug & Sep & Oct & Nov & Dec & Jan & Feb & Mar & Apr & May & Jun \\
\hline$A$ & $\begin{array}{l}\text { F -Torrenticolidae Piersig } 1902 \\
\text { G - Torrenticola Piersig }\end{array}$ & & & & & & & & & & & & \\
\hline 1 & Torrenticola uttarakhandensis & 01 & 02 & 04 & 00 & 11 & 13 & 15 & 13 & 11 & 14 & 12 & 09 \\
\hline 2 & Torrenticola chatterjeei & 00 & 01 & 06 & 05 & 00 & 08 & 10 & 05 & 00 & 00 & 00 & 07 \\
\hline 3 & Torrenticola turkestanica & 00 & 00 & 00 & 00 & 05 & 00 & 07 & 00 & 03 & 00 & 08 & 00 \\
\hline 4 & Torrenticola wonchoeli & 00 & 00 & 00 & 04 & 00 & 00 & 01 & 06 & 03 & 00 & 01 & 00 \\
\hline 5 & Torrenticola tetraporella & 00 & 00 & 00 & 05 & 03 & 04 & 08 & 00 & 02 & 00 & 00 & 00 \\
\hline 6 & Torrenticola semisuta & 02 & 03 & 05 & 21 & 12 & 11 & 13 & 11 & 17 & 16 & 10 & 07 \\
\hline 7 & Torrenticola nana & 00 & 00 & 05 & 06 & 12 & 08 & 09 & 07 & 05 & 07 & 04 & 00 \\
\hline 8 & $\begin{array}{l}\text { Torrenticola kumari } \\
\text { Genus - Monatractides }\end{array}$ & 00 & 02 & 09 & 07 & 10 & 10 & 11 & 10 & 06 & 09 & 08 & 08 \\
\hline 9 & Monatractides garhwaliensis & 00 & 02 & 11 & 10 & 15 & 15 & 18 & 15 & 12 & 10 & 09 & 11 \\
\hline 10 & Monatractides tuzovskyi & 00 & 00 & 09 & 08 & 09 & 14 & 13 & 16 & 15 & 12 & 10 & 08 \\
\hline \multirow[t]{2}{*}{11} & Monatractides oxystomus & 00 & 01 & 05 & 00 & 00 & 00 & 01 & 04 & 00 & 06 & 00 & 00 \\
\hline & Total & 03 & 11 & 54 & 66 & 77 & 83 & 106 & 87 & 74 & 74 & 62 & 50 \\
\hline B & $\begin{array}{l}\text { Family - Sperchontidae } \\
\text { Genus - Sperchon Kramer }\end{array}$ & & & & & & & & & & & & \\
\hline 12 & Sperchon indicus & 03 & 05 & 08 & 15 & 19 & 25 & 27 & 27 & 20 & 18 & 15 & 12 \\
\hline 13 & Sperchon garhwalensis & 02 & 00 & 00 & 12 & 16 & 21 & 28 & 26 & 21 & 20 & 16 & 12 \\
\hline 14 & $\begin{array}{l}\text { Sperchon plumifer } \\
\text { Genus -Sperchonopsis }\end{array}$ & 03 & 04 & 06 & 07 & 06 & 08 & 09 & 00 & 18 & 12 & 10 & 08 \\
\hline 15 & $\begin{array}{l}\text { Sperchonopsis verrucosa } \\
\text { Total }\end{array}$ & $\begin{array}{l}00 \\
08\end{array}$ & $\begin{array}{l}00 \\
09\end{array}$ & $\begin{array}{l}00 \\
14\end{array}$ & $\begin{array}{l}06 \\
40\end{array}$ & $\begin{array}{l}08 \\
49\end{array}$ & $\begin{array}{l}05 \\
59\end{array}$ & $\begin{array}{l}01 \\
65\end{array}$ & $\begin{array}{l}05 \\
58\end{array}$ & $\begin{array}{l}00 \\
59\end{array}$ & $\begin{array}{l}00 \\
50\end{array}$ & $\begin{array}{l}00 \\
41\end{array}$ & $\begin{array}{l}00 \\
32\end{array}$ \\
\hline C & $\begin{array}{l}\text { Family - Hygrobatidae } \\
\text { Genus - Atractides Koch }\end{array}$ & & & & & & & & & & & & \\
\hline 16 & Atractides indicus & 04 & 04 & 12 & 16 & 19 & 21 & 25 & 21 & 18 & 16 & 13 & 11 \\
\hline 17 & Atractides garhwali & 00 & 00 & 05 & 10 & 13 & 14 & 18 & 22 & 17 & 12 & 14 & 09 \\
\hline 18 & Atractides incertus & 00 & 01 & 02 & 06 & 06 & 07 & 02 & 09 & 08 & 00 & 00 & 01 \\
\hline 19 & Atractides panesari & 00 & 00 & 00 & 05 & 00 & 06 & 08 & 04 & 03 & 00 & 00 & 00 \\
\hline 20 & $\begin{array}{l}\text { Atractides ootacamundis (Cook) } \\
\text { Genus - Hygrobates Koch }\end{array}$ & 00 & 00 & 01 & 00 & 06 & 00 & 05 & 00 & 00 & 05 & 00 & 01 \\
\hline 21 & Hygrobates gangeticus & 00 & 01 & 00 & 00 & 00 & 00 & 06 & 00 & 08 & 00 & 00 & 00 \\
\hline \multirow[t]{2}{*}{22} & Hygrobates fluviatilis & 00 & 00 & 01 & 05 & 06 & 10 & 12 & 10 & 08 & 05 & 00 & 04 \\
\hline & Total & 04 & 06 & 21 & 42 & 50 & 58 & 76 & 66 & 62 & 38 & 27 & 26 \\
\hline D & $\begin{array}{l}\text { Family - Aturidae Thor } \\
\text { Genus - Kongsbergia Thor }\end{array}$ & & & & & & & & & & & & \\
\hline 23 & Kongsbergia indica & 02 & 00 & 00 & 06 & 00 & 09 & 10 & 09 & 06 & 03 & 05 & 00 \\
\hline \multirow[t]{2}{*}{24} & Kongsbergia himalayaensis & 00 & 00 & 00 & 06 & 00 & 06 & 09 & 00 & 00 & 00 & 00 & 00 \\
\hline & Total & 02 & 00 & 00 & 12 & 00 & 15 & 19 & 09 & 06 & 03 & 05 & 00 \\
\hline$E$ & $\begin{array}{l}\text { Family - Lebertiidae } \\
\text { Genus - Lebertia Neuman }\end{array}$ & & & & & & & & & & & & \\
\hline \multirow[t]{3}{*}{25} & Lebertia spp. & 00 & 01 & 00 & 00 & 05 & 00 & 08 & 06 & 07 & 04 & 00 & 00 \\
\hline & Total & 00 & 01 & 00 & 00 & 05 & 00 & 08 & 06 & 07 & 04 & 00 & 00 \\
\hline & Total no. of water mites & 17 & 27 & 89 & 160 & 181 & 215 & 274 & 226 & 208 & 169 & 135 & 108 \\
\hline
\end{tabular}

July. In Spot-2, maximum density of 274 units. $\mathrm{m}^{-2}$ was noticed in January, and minimum 17 units. $\mathrm{m}^{-2}$ in July. The highest number (1809) of Hydrachnidia was collected from Spot-2. The species common to both spots were Torrenticola uttarakhandensis, T. wonchoeli, T. nana, $T$. kumari, $T$. semisuta, Monatractides garhwaliensis and M. oxystomus (family Torrenticolidae Piersig 1902), Sperchon indicus, S. garhwalensis (family Sperchontidae Thor, 1900), Atractides indicus, A. garhwali, A. incertus, A. ootacamundis and Hygrobates fluviatilis (Cook) (family Hygrobatidae). However, species limited to Spot-1 were $T$. muranyii, $M$. kontschani (family Torrenticolidae Piersig 1902) and
Feltria gereckei, F. indica (family Feltriidae K.Viets, 1926), and species limited to Spot-2 were T. chatterjeei, T. turkestanica, T. tetraporella and M. tuzovskyi (family Torrenticolidae Piersig 1902), S. plumifer, Sperchonopsis verrucosa (Sperchontidae Thor, 1900), A. panesari, Hygrobates gangeticus (family Hygrobatidae), Kongsbergia indica, K. himalayaensis (Aturidae Thor, 1900) and Lebertia (family Lebertiidae),

Shannon's diversity index ranged from 1.67 to 2.73 in Spot-1 and 1.87 to 2.99 in Spot-2. The maximum diversity (2.73) was observed in January and the least diversity (1.67) was noticed during July month in Spot-1, whereas in Spot-2, upper limit of diversity index (2.99) 

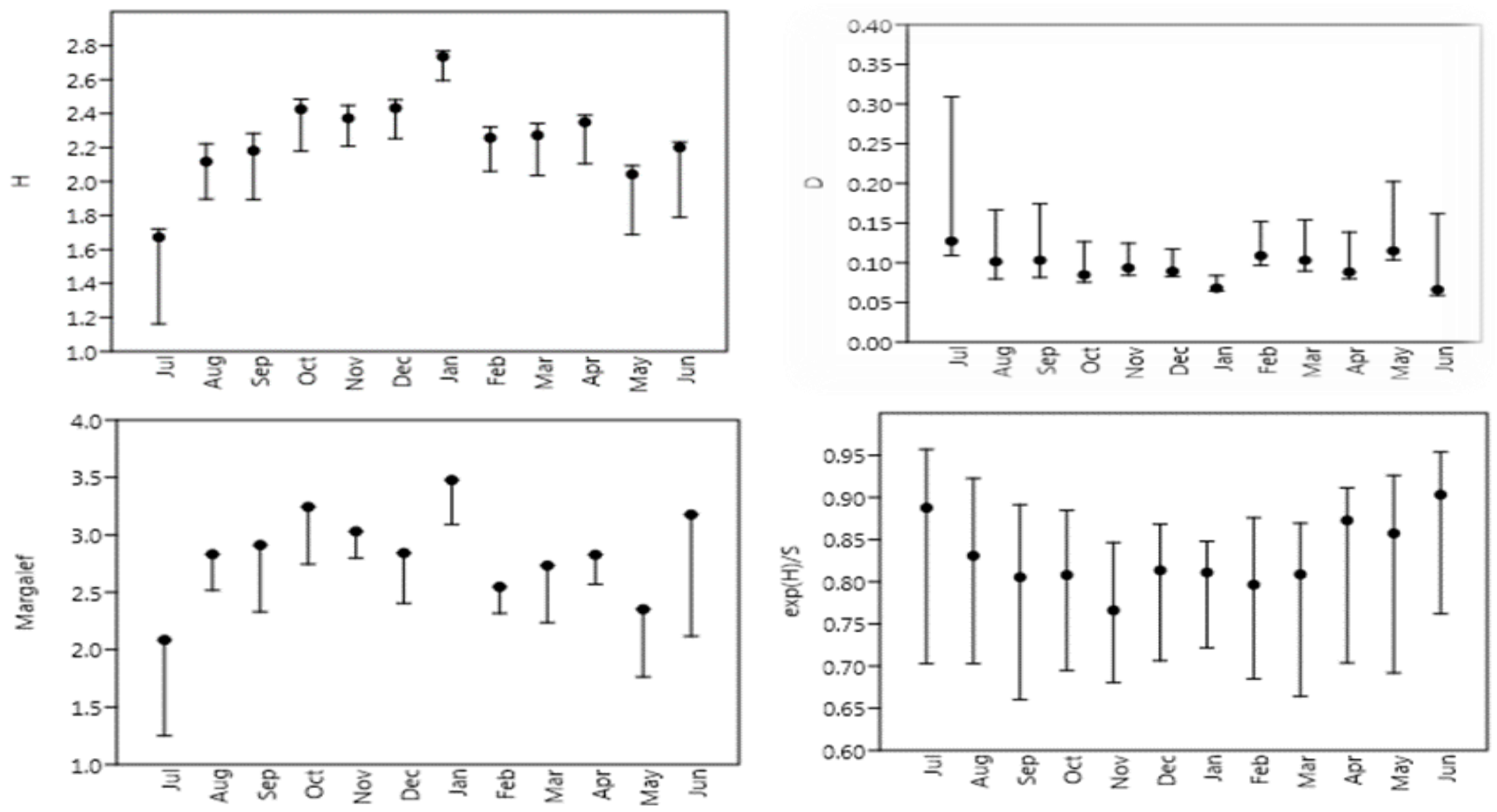

Fig. 2. Shannon diversity index, Simpson index, Margalef species richness index and Pielou index, respectively for Spot-1 of Khankra gad during 2018-20.
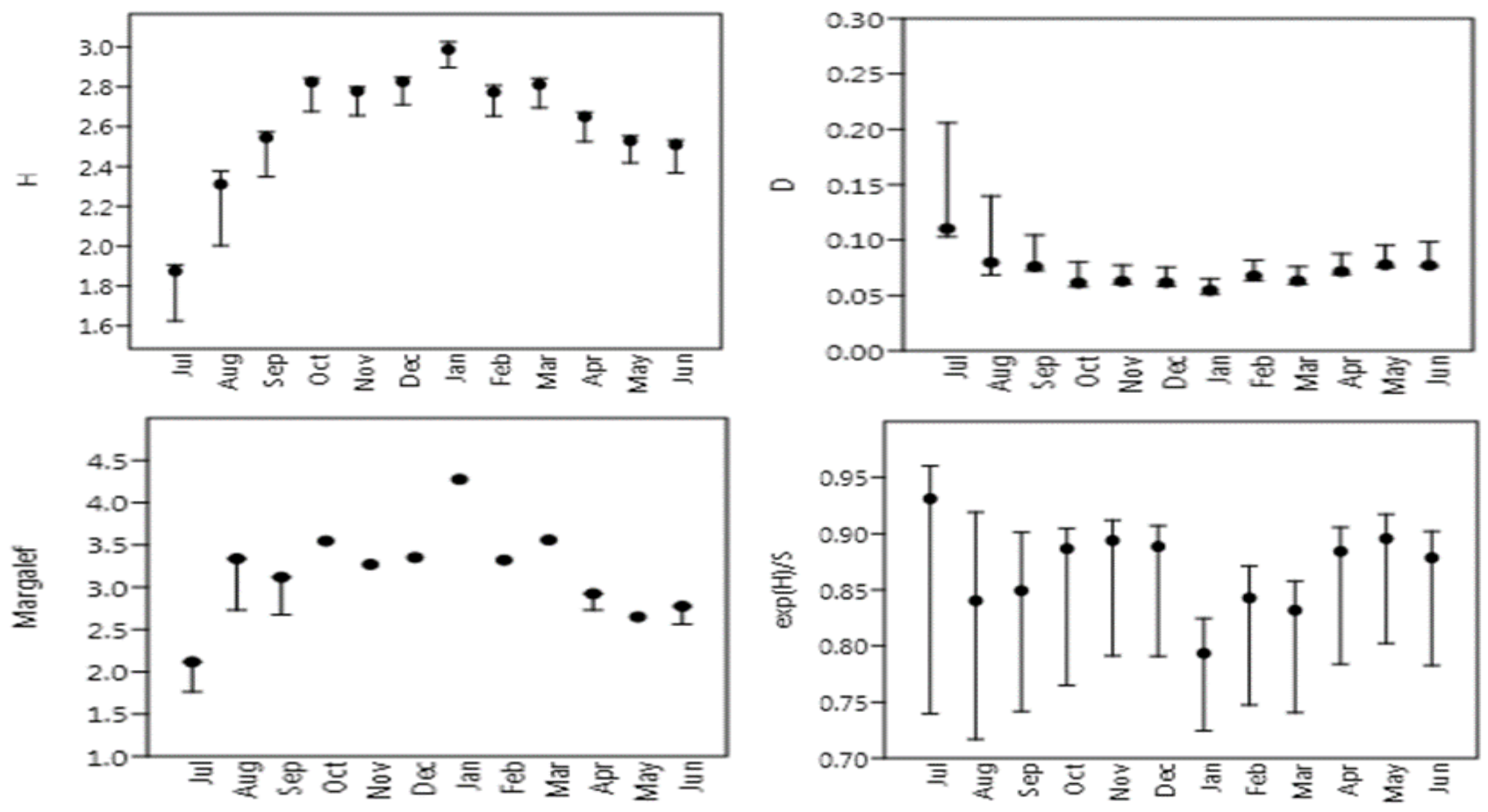

Fig. 3. Shannon diversity index, Simpson index, Margalef species richness index and Pielou index, respectively for Spot-2 of Khankra gad during 2018-20.

was observed in January and lower (1.87) during July. Pielou's index $(\mathrm{J} ')$ was calculated for evenness which also showed variations similar to Shannon diversity index. Higher evenness (0.95) was in June and lower evenness $(0.75)$ in the October month in Spot-1. At Spot-2, higher evenness (0.93) was recorded in July and lower (0.79) in January (Fig.2 and Fig.3).
The value of Margalef species richness index (d) was maximum in January at both the spots (3.47 at Spot-1 and 4.27 in Spot-2). The minimum value was observed in July at both the spots (2.08Spot-1 and 2.11 at Spot2). The Simpson index (D) was maximum (0.12) at Spot-1 and 0.11 at Spot-2. Simpson Index was lowest $(0.06)$ in June in Spot-1 and (0.05) in January in Spot-2 
Negi, S. et al. / J. Appl. \& Nat. Sci. 13(1): 258 - 267 (2021)

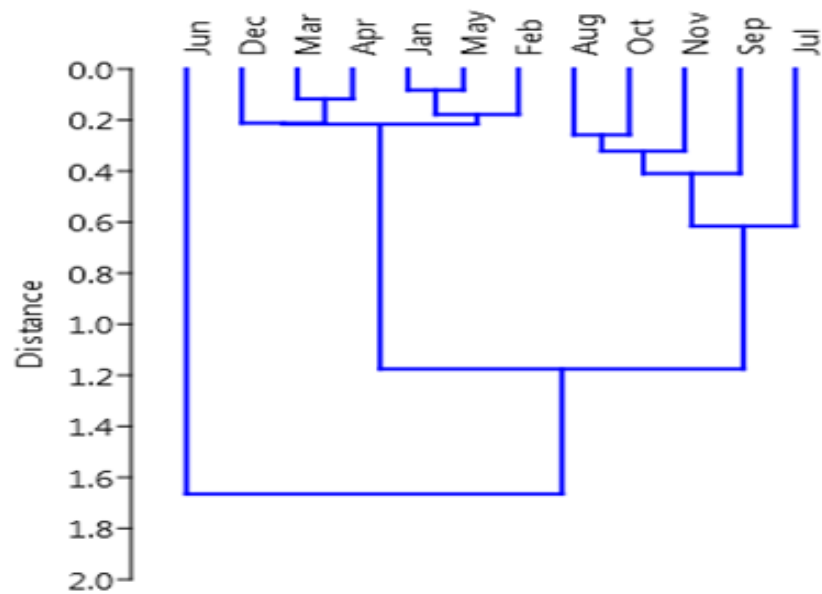

Fig. 4. Hierarchy clustering between water mite taxa of different months during the year 2018-20 at the Spot-1 Khankra gad.

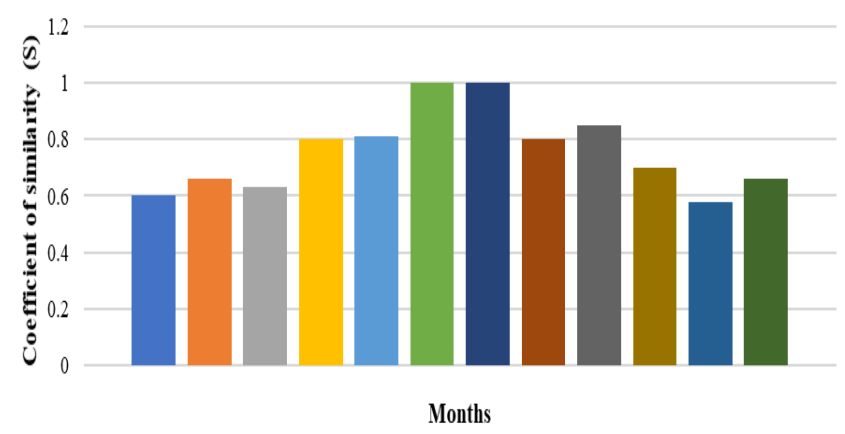

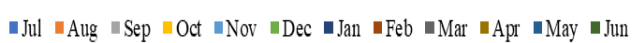

Fig. 6. Coefficient of Similarity (S) between two Spot-1 and Spot-2 of Khankra gad during different months 2018-20.

(Fig.2 and Fig.3). The similarity in taxa of water mites during different months is analysed by using cluster analysis and is present in Fig. 4 for Spot-1 and Fig. 5 for Spot-2, respectively. Variability can be seen through Fig.6, which depicts that in Spot-1 and Spot-2, it is higher in favourable months January (1) and February (1), and lowest in May (0.58).

The Pearson correlation analysis of species abundance of water mites with some physicochemical parameters and linear regression plots for Spot-1 and Spot-2 were drawn (Fig.7 and Fig. 8). For Spot-1 and Spot-2, there was a strong negative linear relationship between the abundance of water mites and water temperature $(r=-$ $0.78,-0.95$, respectively). There was a positive linear relationship between the abundance of water mites and $\mathrm{pH}$ of water for Spot-1 and Spot-2 ( $r=0.59,0.37$ respectively). For Spot-1 and Spot-2, a negative linear regression relationship was noted between the abundance of water mites and water velocity $(r=-0.69$, 0.91 , respectively). There was a significant positive linear relationship between the abundance of water mites and dissolved oxygen of water for Spot-1 and

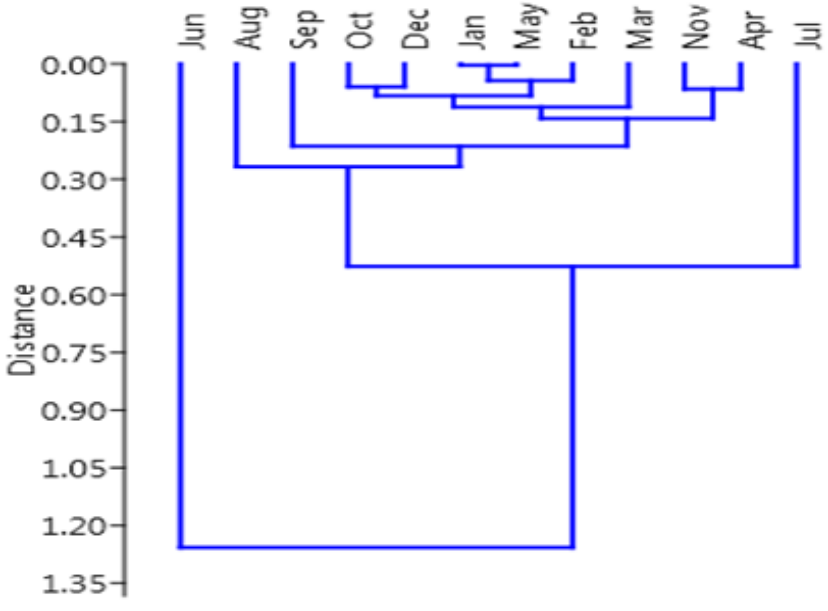

Fig. 5. Hierarchy clustering between water mite taxa of different months during the year 2018-20 at the Spot-2 Khankra gad.

Spot-2 ( $r=0.72,0.64$ respectively).

A positive linear regression relationship was also observed between the abundance of water mites and total alkalinity ( $r=0.68,0.86$ respectively). There was a positive linear relationship between the abundance of water mites and total hardness of water for Spot-1 and Spot-2 ( $r=0.41,0.91$ respectively).

\section{DISCUSSION}

Aquatic mites are among the most taxonomically diverse group of the Acari in freshwater but comparatively less studied for their population dynamics. In the present study, we observed that at Spot-2, which is in the lower reach of stream, there is high density (274units.m ${ }^{-2}$ ) and diversity (25species) in comparison to Spot-1, which is the upper reach (maximum density-177 units. $\mathrm{m}^{-2}$ and 19 species). Our observations suggest that species diversity increased with species richness and was highest in January in both sampling spots. Similar results have been reported by Bahuguna et al. (2019) in the spring-fed Randi Gad stream of Garhwal Himalayas. They reported a total of fourteen species belonging to five families. A maximum number of 138 mites $\mathrm{m}^{-2}$ was recorded in January and a minimum density of 03 units. $\mathrm{m}^{-2}$ in July. Bahuguna et al. (2019) opined that the highest number of aquatic mites observed in the winter season might be correlated with moderate to high periphyton growth in Randi Gad of Garhwal Himalaya. Water mite species tend to increase with increasing amounts of nutrients and ideal environmental conditions. The improved homogeneity of range and species of water mites indicates excellent water quality (Abhijan et al., 2013). Di Sabatino et al. (2000) found that water temperature is an important factor for the assemblage of Hydrachnidia community and affects the latitudinal and altitude distribution pattern of the 


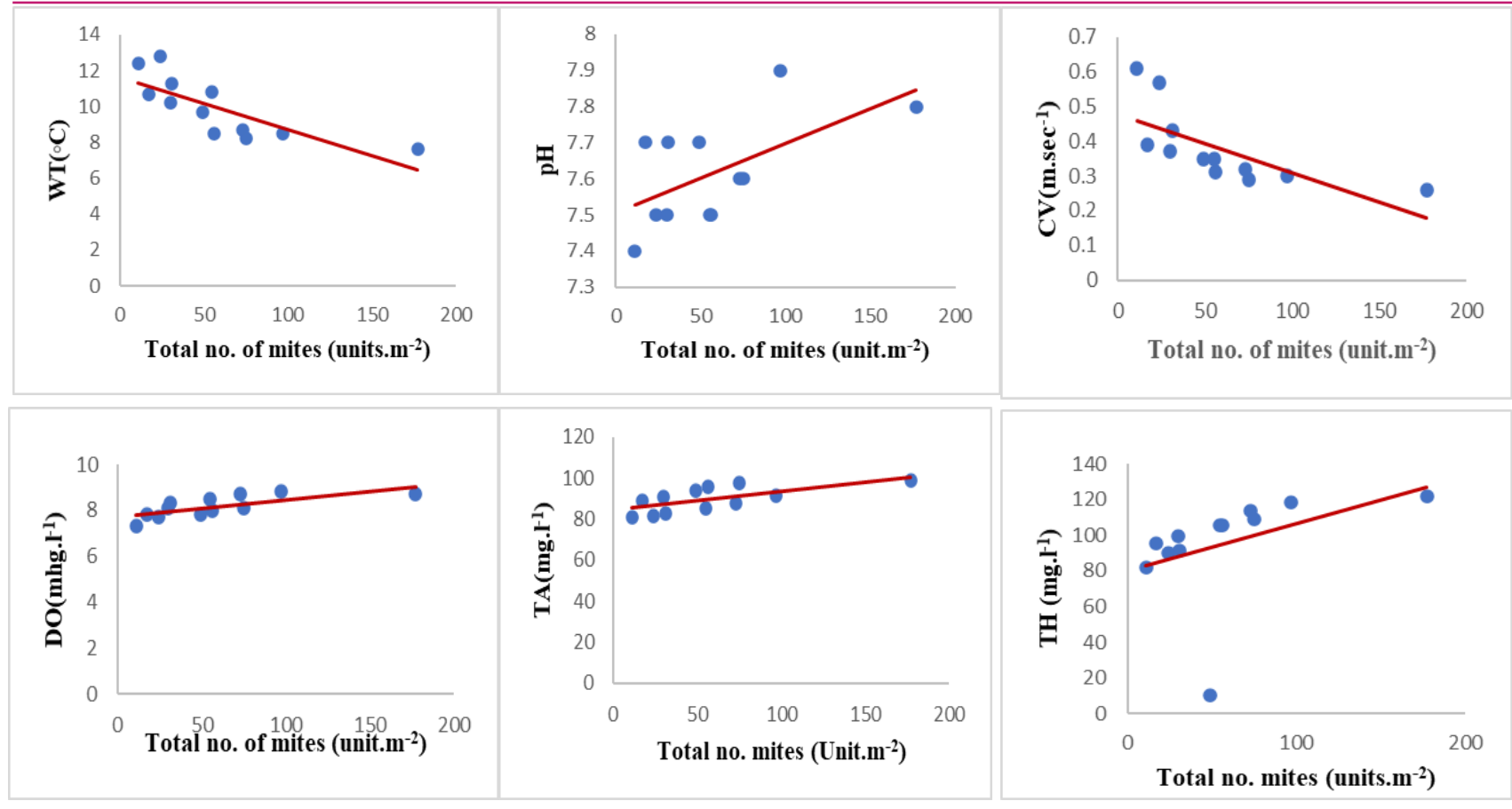

Fig. 7. Regression plot of water mite species abundance against physicochemical parameters of Khankra gad at Spot-1.
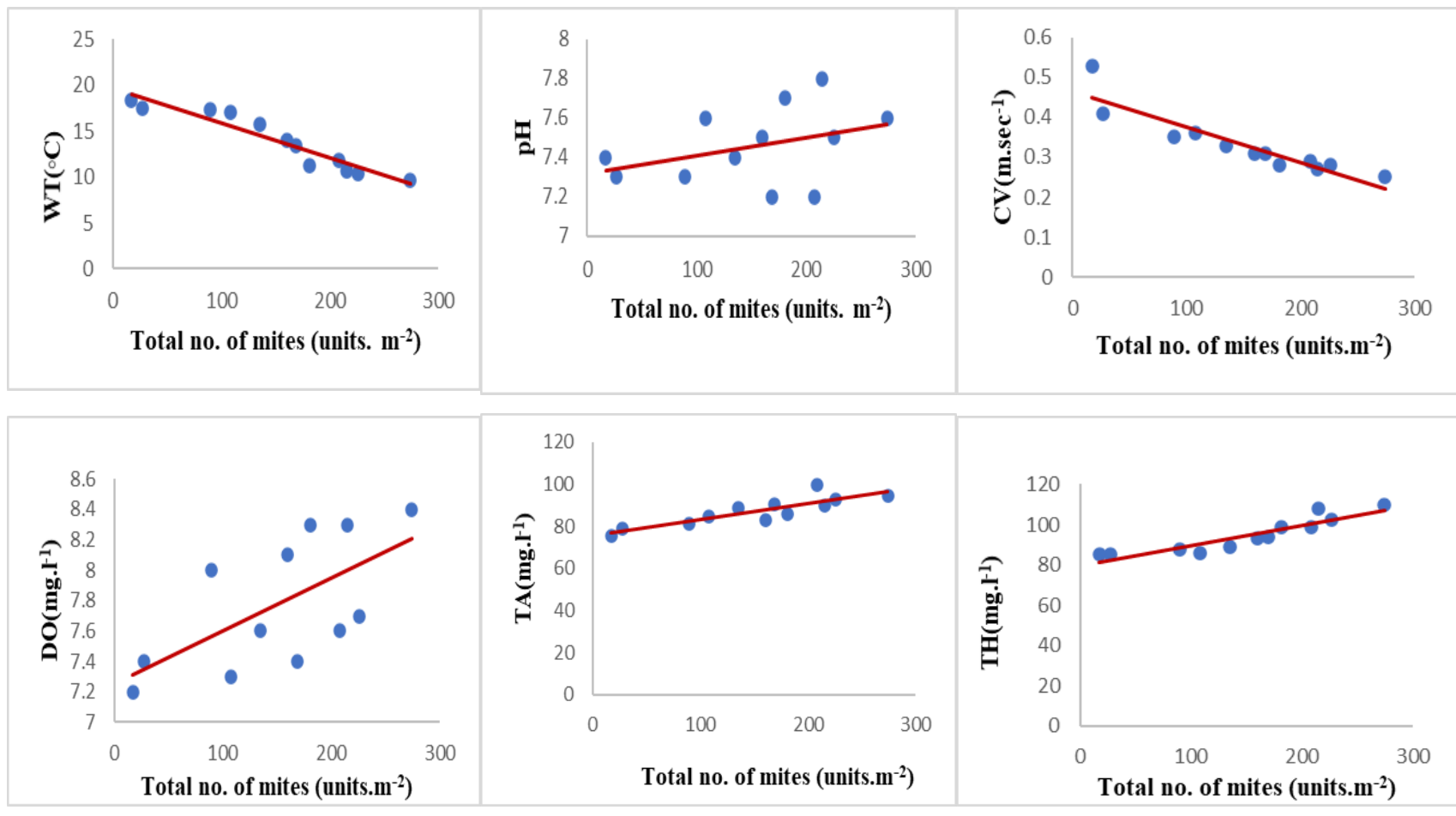

Fig. 8. Regression plot of water mite species abundance against physicochemical parameters of Khankra gad at Spot-2.

Hydrachnidia community. Smit and van der Hammen (2000) stated that other factors such as $\mathrm{pH}$ of water, DO, water velocity, and other ecological parameters can also influence the formation of Hydrachnidia communities.

It was observed that the stream velocity variation was also responsible for fluctuation in population density and diversity of water mites. The velocity of the Khankra gad varied from an average of 0.21 to $0.25 \mathrm{~m} . \mathrm{sec}^{-1}$ with the highest flow of $0.61 \mathrm{~m} . \mathrm{sec}^{-1}$ ) at Spot-1 and the lowest flow of $0.25 \mathrm{~m} . \mathrm{sec}^{-1}$ at Spot-2. The average water temperature showed higher value at Spot-2 and a lower value at Spot-1. The variation in the water temperature range was due to the difference in solar radiation caused by the canopy and riparian vegetation around the spots. The study by Thani and Phalaraksh (2008) showed that the difference in the water temperature in a river or stream depends on the climate as well 
Negi, S. et al. / J. Appl. \& Nat. Sci. 13(1): 258 - 267 (2021)

as on the time of sampling and the amount of sunlight. DO value fluctuated between $8.8 \pm 0.3 \mathrm{mg}^{-1}$ and $7.2 \pm 0.1 \mathrm{mg} . \mathrm{I}^{-1}$. An increase in DO value during winter and a decrease in summer are well-known characteristic features of the freshwater ecosystem. $\mathrm{pH}$ values were found to vary between 7.2 to 7.8. This observation corroborates with the work of Malik et al. (2012), who recorded $\mathrm{pH}$ values 7.09 to 8.03 in Asan reservoir. There was a high range of total alkalinity $81.2 \pm 0.8 \mathrm{mg} . \mathrm{I}^{-}$ ${ }^{1}$ to $99.9 \pm 0.5 \mathrm{mg}^{-1}{ }^{-1}$ in the present study. The hardness value was also high $109.8 \pm 13.2 \mathrm{mg}^{-l^{-1}}$. Das and Das (1997) stated that productive water should have hardness above $20 \mathrm{mg} . \mathrm{I}^{-1}$. In view of this statement, Khankra gad was a highly productive one. Free $\mathrm{CO}_{2}$ during the present study was absent. Several factors such as water temperature, current velocity, vegetation, substratum, dissolved substances, food, competition between species etc., regulate the occurrence and distribution of stream invertebrates as studied by Hynes (1970).

While observing the impact of the certain ecological detrimental factor on the density of water mites, it was observed that there was a negative correlation between mite density vs water temperature (Spot-1 $r=-0.78$, Spot-2 $r=-0.95$ ) and water velocity (Spot-1 $r=-0.69$, Spot-2 = -0.91) which indicated that temperature and velocity was not a detrimental factor for mites density here. The dissolved oxygen, $\mathrm{pH}$, total alkalinity and total hardness showed a positive correlation with the mites density in both streams. As can be understood, the total alkalinity favours periphyton growth in moderately flowing hill streams, and thereby it helps in increasing mites population during the winter months. Similar results have been noticed by Kumar and Dobriyal (1993) for the benthic diversity of Garhwal Himalayan hill streams. Baluni et al. (2018) reported that the periphyton was maximum in January and minimum in August from the Khankra gad stream.

A comparison of the similarities between the upstream and downstream catchment sites showed a similar pattern of cluster formation. In December and January, the similarity was highest (1) for both the sampling spots. These findings suggested that the establishment of a certain type of fauna at sampling sites is influenced by the occurrence of certain types of water bodies and particular types of landscape and physicochemical parameters (Stryjecki et al., 2016).

The water quality of the stream in the present study was observed very good and unpolluted. Generally, freshwater ecosystems are threatened by pollution, which affects the physicochemical properties and causes the degradation of aquatic biodiversity. In assessing the diversity of aquatic species, water quality plays an important role. (Allan and Flecker, 1993). The $\mathrm{pH}$ of the water is also known to affect the population level of aquatic organisms; therefore, this variation in water parameters greatly affects the distribution pattern of these aquatic insects (Popoola and Otalekor, 2011).

\section{Conclusion}

The present study concluded that Hydrachnidia density and diversity decreased with increasing elevation. A firm spatial and temporal gradient was displayed in the water mite habitat, and their parasitic lifestyle combines a strong dependence on habitat in response to dispersal change. Various water parameters of our study indicate that Khankra gad water can serve as a good habitat for many aquatic organisms, including mites. Therefore, our baseline survey addresses the need for more in-depth studies along the stream's entire length to explore the further possibility of biodiversity addition as new niche-specific species.

\section{ACKNOWLEDGEMENTS}

Authors (S.N) and (P.B) gratefully acknowledge the financial support granted by the Science and Engineering Research Board (SERB) under a major project No. ECR /2016/001291.

\section{Conflict of interest}

The authors declare that they have no conflict of interest.

\section{REFERENCES}

1. Abhijna,U. G., Ratheesh, R. and Biju Kumar, A. (2013). Distribution and diversity of aquatic insects of Vellayani lake in Kerala. Journal of Environmental Biology, 34(3), 605-611.

2. Allan, J.D. and Flecker, A.S. (1993). Biodiversity conservation in running waters. Bioscience, 43, 32, 43.

3. APHA (2012). Standard methods for examination of Water and Wastewater. 22nd Edi., APHA, AWWA, WPCF, Washington D.C., USA.

4. Bahuguna, P., Negi, S. (2020). A checklist of the water mites (Acari: Hydrachnidia) of Garhwal Himalaya with some new records. (Edited by R. A. Singh, Conference Book on Geology and Natural Resource of Himalaya. A.S.R. Publication, Lucknow -226008.

5. Bahuguna, P., Negi, S., and Dobriyal, A. K. (2019). Density and diversity of aquatic mites in a spring fed stream of Garhwal Himalaya, India. J. of Mountain Res., 14(2), 5559. https://doi.org/10.51220/jmr.v14i2.6

6. Bahuguna, P., Rana, K. K., Rayal, R., and Khanduri, N. C (2020). Density and diversity of aquatic mites in a glacierfed river Mandakani from Garhwal Central Himalaya, India. Uttar Pradesh Journal of Zoology, 41(10), 1-8.

7. Bahuguna, Pankaj and Dobriyal, A. K. (2020). Population structure and drifting pattern of aquatic mites in Randi Gad, a tributary of River Alaknanda in Garhwal Himalaya, Uttarakhand, India. J. Mountain. Res., (15), 63-70. https:// doi.org/10.51220/jmr.v15i1.7 
8. Baluni, P., Kumar, R. and Joshi, H.K. (2018). Ecology, distribution pattern, density and diversity of periphyton in Khankra spring fed stream of Garhwal Himalaya, India. J. Mountain. Res.,13, 95-99.

9. Cook, D. R. (1967). Water mites from India. Memoirs of the American Entomological Institute, 9: 1-411.

10. Cook, D. R., and R. D. Mitchell (1953). Notes on collecting water-mites. Turtox News, 30,122-125.

11. Cook, D.K. (1974). Water Mites Genera and Subgenera. American Entomological Institute, 21, 860.

12. Das, M.K. and Das R.K., (1997). Fish and Prawn diseases in India, diagnostic and control. Journal of Inland fisheries society of India. Barrackpore, pp: 139.

13. Di Sabatino A., Gerecke R., Martin P. (2000). The biology and ecology of lotic water mites (Hydrachnidia). Freshwater Biology, 44,47-62. https://doi.org/10.1046/.1365-242 7.2000.00591.x

14. Gerecke R (2003). Water mites of the genus Atractides Koch, 1837 (Acari: Parasitengona: Hygrobatidae) in the western Palaearctic region: a review. Zoological Journal of the Linnean Society, 138, 141-378. https://doi.org/10.1 046/j.1096-3642.06-0.00051.x

15. Hynes, H. B. N. 1970. The Ecology of running waters. Liverpool University Press. 543 pp.

16. Kumar, N. and Dobriyal, A. K. (1992). Some observations on the water mites of a Hill stream Khandagad in Garhwal Himalaya. Journal of Freshwater Biology, 4, 193-197.

17. Kumar, N. and Dobriyal, A. K. (1993). Benthic diversity of Garhwal Himalayan hill streams in relation to their fishery potential. The third Indian fisheries forum proceedings, Pant Nagar. pp 159- 162.

18. Kumar, N., Kumar, K., Kumar, S. and Pesic, V. (2006) Monatractides tuzovskyi sp. nov. (Acari: Torrenticolidae), a new water mite species from the Garhwal Himalayas (India). Acarina, 14(2), 81-83.

19. Kumar, N., Kumar. K. and Pesic, V. (2007). Two new species of Sperchon Kramer (Acari: Hydrachnidia: Sperchontidae) from the Garhwal Himalayas (India). Systematic and Applied Acarology, 12, 31-36. https://doi.org/1 $0.11158 /$ saa.12.1.5

20. Lundblad, O. (1934). Report on Hydracarina (Yale North India Exped. 7). Memoirs of the Connecticut Acad-emy of Arts and Science, New Haven, 10, 85-118.

21. Malik, D.S., Kumar S. and Nagar, S. (2012). Seasonal nutrient characteristics of Asan reservoir at Dehradun, Uttarakhand, J. Sustainable Environ. Res., 1(2), 233-238.

22. Margalef, R. (1958). Temporal succession and spatial heterogeneity in phytoplankton in perspectives in Marine Biology, Buzzati-Traverso (ed.) Univ. Calif. Press, Berkeley, pp. 323-347.

23. Martin. P., (2008). Water mites (Hydrachnidia, Acari) and insects: a survey of a seldom considered relationship. Entomol Heute, 20,45-75.

24. Mitchell, R.D. and Cook, D.R. (1952). The preservation and mounting of water-mites. Turtox News, 30(9).

25. Pesic, V. and Panesar, A. (2008). Studies on water mites (Acari, Hydrachnidia) from the Himalayas, I. The water mite genus Feltria Koenike, with descriptions of eight new species. Zootaxa, 1758, 1-28. https://doi.org.10.11646/ zootaxa.2119.1.1

26. Pesic, V., Chatterjee, T., Das, M. K. and Bordoloi, S. (2012). Two rare water mite species (Acari, Hydrachnidia) from the streams of the Indian eastern Himalayan region. Systematic and Applied Acarology, 17(4): 458-464. https://doi.org/10.11158/saa.17.4.15

27. Pesic, V., Kumar, N. and Kumar, K. (2007a). Two new species of water mites of the family Hygrobatidae (Acari: Hydrachnidia) from the Garhwal Himalayas (India). Systematic and Applied Acarology, 12, 161-166. https:// doi.org/10.11158/saa/12.2.11

28. Pesic, V., Kumar, N. and Kumar, K. (2007b). A new species of Monatractides (Acari: Hydrachnidia: Torrenticolidae) and new records of other torrenticolid water mites from the Garhwal Himalayas (India). Systematic and Applied Acarology, 12(3-4), 205-212. https:// doi.org/10.11158/saa.12.3.5

29. Pesic, V., Smit, H. and Bahuguna, P. (2019a). New records of water mites (Acari: Hydrachnidia) from the Western Himalaya with the description of four new species. Systematic and Applied Acarology, 24(1), 59-80. https:// doi.org/10.11158/saa.24.1.5

30. Pesic, V., Smit, H. and Bahuguna, P. (2019b). New records of water mites (Acari: Hydrachnidia) from the Western Himalaya and description of three new species from Asia. Systematic and Applied Acarology,24(10),18681880. 20. https://doi.org/10.11158/saa.25.10.6

31. Pesic, V., Smit, H. and Bahuguna, P. (2020a). A new species of Kongsbergia from the Western Himalaya with a key to the species of the genus of India (Acari: Hydrachnidia). Ecologia Montenergrina, 27, 35-38. 21.

32. Pesic, V., Smit, H. and Bahuguna, P., Negi, S., Dobriyal, A. K. (2020b). Torrenticolid water mites of India with description of three new species (Acari: Hydrachnidia, Torrenticolidae), Systematic and Applied Acarology,25(2), 255-267. https://doi.org/10.11158/saa.25.2.7

33. Pielou, E. C., (1966). The measure of diversity in different types of biological collections. J Theoret. Biol., 13, 131-144.

34. Popoola, KOK., Otalekor, A. (2011). Analysis of aquatic insects' communities of Awba reservoir and its physicochemical properties. Research Journal of Environmental and Earth Sciences,3(4), 422-428.

35. Prasad, (1974). A Catalogue of mites of India. Ludhiana, Indira Acarology Publishing House, $320 \mathrm{pp}$.

36. Shannon, C. E. and W. Wiener, (1949). The mathematical theory of communication. Urbana, University of Illinois Press, 177pp.

37. Simpson, E. H. (1949). Measurement of diversity. Nature, 163 (4148), 688.

38. Smit H, van der Hammen $H$ (2000) Atlas of the Dutch water mites (Acari: Hydrachnidia) (in Dutch). Nederlandse Faunistische Mededelingen, 13,1-266

39. Stryjecki, R., Zawal, A., Stępień, E. et al. (2016). Water mites (Acari, Hydrachnidia) of water bodies of the Krąpiel River valley: interactions in the spatial arrangement of a river valley. Limnology, 17, 247-261. https:// doi.org/10.1007/s10201-016-0479-6

40. Thani, I and Phalaraksh, C. (2008). A Preliminary Study of Aquatic Insect Diversity and Water Quality of Mekong River, Thailand. KKU Sci. J., 6 (Supplement), 95-106.

41. Walter, C. (1928). Zur Kenntnis der Mikrofauna von Britisch Indien. II. Hydracarina. Records of the Indian Museum, Calcutta, 30(1), 57-108.

42. Welch, P.S (1948). Limnological Methods. Blackiston Co. Philadephia. 